livraisons

d'Histoire

de l'Architecture

\section{Livraisons de l'histoire de l'architecture}

$21 \mid 2011$

Bâtir et orner

\title{
Terminer le palais du Louvre, 1861
}

Completing the Louvre palace, 1861

Den Bau des Louvre Palastes beenden, 1861

\section{Cécile Souchon}

\section{OpenEdition}

\section{Journals}

Édition électronique

URL : http://journals.openedition.org/lha/273

DOI : $10.4000 /$ lha. 273

ISSN : 1960-5994

Éditeur

Association Livraisons d'histoire de l'architecture - LHA

Édition imprimée

Date de publication : 10 juin 2011

Pagination : 59-62

ISSN : 1627-4970

Référence électronique

Cécile Souchon, «Terminer le palais du Louvre, 1861 », Livraisons de I'histoire de l'architecture [En ligne], 21 | 2011, mis en ligne le 10 juin 2013, consulté le 30 avril 2019. URL : http://journals.openedition.org/ Iha/273; DOI : 10.4000/lha.273

Ce document a été généré automatiquement le 30 avril 2019

Tous droits réservés à l'Association LHA 


\title{
Terminer le palais du Louvre, 1861
}

\author{
Completing the Louvre palace, 1861 \\ Den Bau des Louvre Palastes beenden, 1861
}

\section{Cécile Souchon}

1 Depuis que la République s'est fermement ancrée dans les institutions françaises, dans nos livres et leçons d'histoire, dans nos habitudes mentales ou nos convictions politiques, nous vivons dans une capitale quelque peu « décalée » : Pour nous, en effet, le Louvre est un musée, le palais royal, un quadrilatère ministériel et luxueux, le palais Bourbon, un lieu de réunion démocratique, et les Tuileries, un grand jardin public, pour ne citer que quelques exemples.

2 Ce n'est, en tous cas, pas la ville qu'évoque le beau fonds d'archives de l'Agence d'architecture du Louvre et des Tuileries (64 AJ) versé aux Archives nationales en plusieurs fois depuis 1971 grâce à l'opiniâtreté d'Emmanuel Jacquin et l'aide de nombreux correspondants et complices, impossibles à citer tous.

370000 pièces environ composent cet ensemble, réparti en 922 cotes plus ou moins complexes, pour prendre en compte aussi bien les registres et cartons d'archives écrites administratives (correspondance, rapports, devis...), que les portefeuilles d'archives graphiques et documents figurés (dessins, calques, plans, coupes, élévations, projets, détails...), les plans roulés (grands formats), et plus de 5000 photographies de grand intérêt sur le chantier et les décors. Le tout se trouve dans l'état matériel que connaissent des documents d'archives d'usage constant et de manipulation malaisée, lorsqu'ils touchent à un grand ensemble de bâtiments des plus prestigieux, puisqu'ils abritèrent encore pendant une bonne partie du XIX ${ }^{e}$ s., ceux qui incarnaient le Pouvoir au sein du paysage urbain qui est si familier aux Parisiens.

4 «Il m'apparait, dit Louis-Napoléon, que le palais du Louvre ne doit pas rester dans un état qui déshonore le Gouvernement, et il est souhaitable que du travail soit fourni aux classes laborieuses. J'ai par conséquent conçu le projet de l'achèvement de ce palais, dont je souhaite que vous vous chargiez ", déclare celui qui deviendra Napoléon III (l'Empire est proclamé le 2 décembre 1852) à Visconti, son architecte officiel ${ }^{1}$, en 1852. 
Sous cette impulsion décisive, l'agence d'architecture du Louvre et des Tuileries fusionne l'agence des grands travaux du Louvre (Vieux Louvre) confiée à Félix Duban, l'agence de la réunion des Tuileries au Louvre créée en mai 1852, et l'agence des Tuileries conduite par Visconti depuis octobre 1852. La première pierre du Nouveau Louvre est bénie et posée le dimanche 25 juillet 1852, le palais existant, demeure du premier Empereur et du dernier roi de France étant occupé, de préférence à l'Élysée, par le Prince-président depuis février 1852.

6 Visconti meurt brutalement en 1853. À compter de 1854, la responsabilité des travaux passe à Hector Lefuel ${ }^{2}$, qui décède sous la III ${ }^{e}$ République alors que son œuvre a vu le jour, mais a également dû résister aux assauts de la Commune, à la destruction des bureaux de Fontaine en 1848, à l'incendie de la bibliothèque du Louvre en mai 1871.

7 L'inventaire du fonds (300 pages imprimées) publié en 2006 ne porte évidemment pas de jugement sur la qualité des pièces conservées, qu'il s'agisse de grands projets généraux de conception, de détails de tuyauterie, d'échafaudages, de charpente, ou d'éléments du décor. Du coup, sa version « allégée » mise à la disposition du public ne rend pas toujours justice à la splendeur de certaines pièces, fondues dans un recueil aussi peu loquace que possible pour qu'il ne devienne pas illisible par excès de détails.

On peut s'en rendre compte en examinant par exemple « l'étude de façade des bâtiments sur le quai entre le pavillon de Flore et le milieu de la galerie du Bord-de-l'eau » (64 AJ 583 / 6, calque aquarellé entoilé après restauration de $3,80 \mathrm{~m}$ x 0,73) due à Hector Lefuel en $1861^{3}$.

De même que l'on parle pour un orgue de « tuyaux de montre », ceux que peut admirer le public, de même peut-on parler d'un document « de montre " pour cette représentation du déroulement à venir des façades du Louvre le long de la Seine (ill. 1).

De la volonté politique de Napoléon III sont nés sans attendre une approbation des commissions de contrôle comme le conseil des bâtiments civils, puis un financement (qui a coupé court aux discussions), puis moins de dix plus tard, une réalisation. Ce document nous montre à quel point le palais du Louvre enfin déployé en impose, tant dans l'espace occupé que dans l'harmonie des formes, l'intelligence du raccord avec l'existant (jardin des Tuileries) et l'embellissement des berges du fleuve.

11 Rendre les visiteurs du monde entier jaloux des beautés de Paris, susciter leur admiration et leur respect, est un rêve qu'avait déjà caressé Napoléon ${ }^{\mathrm{er}}$ lorsqu'il envisageait en 1812 l'installation du grand dépôt des Archives de l'Empire près du pont d'Iéna et du palais du Roi de Rome.

III. 1 : «Étude de façade des bâtiments sur le quai entre le pavillon de Flore et le milieu de la galerie du Bord-de-l'eau »

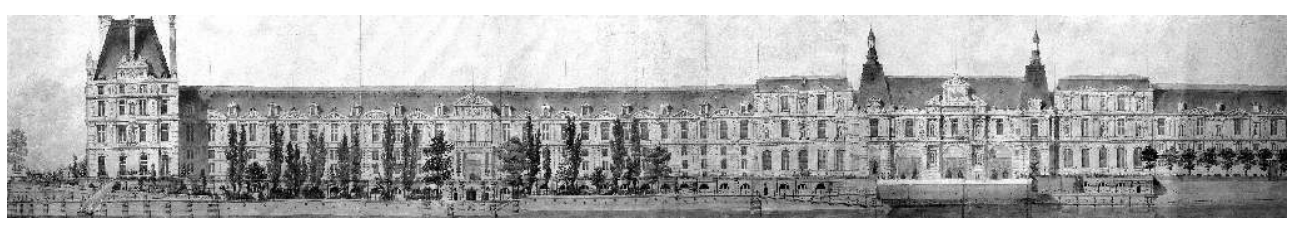

Calque aquarellé entoilé après restauration, 3,80 m x 0,73, Hector Lefuel, 1861. Arch. nat., 64/ AJ $/ 583 / 6$

Cl. Martine Plouvier. 
Ce document, parmi la foule des autres documents de ce fonds d'archives, porte témoignage d'une ardente volonté du Souverain de bâtir, d'orner, et d'y réussir : il s'agit de terminer en beauté (et avec des mesures sociales, qui plus est) ce Louvre des Rois de France, laisser des pierres marquer la Ville pour plus longtemps encore que les hommes illustres, et proposer ce défi à un homme de l'art, un architecte qui soit, et qui sera capable de donner une réalité à ce rêve souvent renouvelé.

\section{NOTES}

1. . Louis Joachim Tullius Visconti, né à Rome (1791) et mort à Paris (1853), français depuis 1799, est petit-fils du créateur du musée du Vatican, et fils du conservateur des Antiquités et tableaux du musée du Louvre. Élève de Percier à l'École des Beaux-Arts (1808-1817), il est second grand prix de Rome en 1814 (architecture). Entre autres réalisations qui le rapprochent de son commanditaire, il conçoit les décorations pour le retour des cendres de Napoléon I $^{\text {er }}(1840)$ et construit le tombeau de l'Empereur aux Invalides.

2. . Hector Lefuel (1810-1880), élève de Huyot aux Beaux-Arts (1829), pensionnaire à Rome (1840-1844), architecte du château de Meudon (1848) de la manufacture de Sèvres (1852), du théâtre du château de Fontainebleau (1853-1855), est appelé au Louvre pour l'achever. Voir Françoise Heilbrun et Geneviève Bresc, Le Photographe et l'architecte. Édouard Baldus, Hector-Martin Lefuel et le chantier du Nouveau Louvre de Napoléon III, Paris, RMN, 1995, 135 p.

3. Voir l'inventaire imprimé de $64 \mathrm{AJ}$, p. 177 : le document reproduit (64 AJ 582/8) est de la même veine que celui dont il est question ici.

\section{RÉSUMÉS}

Le prince-président bientôt empereur Napoléon III rêve, comme son illustre prédécesseur, de marquer Paris, sa capitale, pour continuer d'embellir la ville et pour sa propre gloire, ainsi que pour donner du travail aux classes fragiles. Le palais du Louvre, l'un des sièges du pouvoir, est au cœur de Paris un chantier inachevé au moins depuis le règne d'Henri IV. Mobilisant des architectes renommés, Félix Duban, Louis Visconti, puis Hector Lefuel, leur apportant son attention personnelle et des crédits importants, Napoléon lance dès 1853 les travaux d'achèvement du Louvre, de sa jonction avec les Tuileries, et de la décoration de ces palais au goût de l'époque. Parmi les milliers de documents déposés aux Archives nationales par l'agence d'architecture du Louvre et des Tuileries, le développé de la façade du Louvre donnant sur la Seine, calque aquarellé réalisé sous l'autorité de Lefuel, souligne l'ampleur du projet et la splendeur que l'on en attend pour les quais de Paris.

Napoleon III, prince-president soon to be emperor dreams to leave his mark on Paris, his capital, as his notorious predecessor did. He wishes to do so in order to keep on embellishing the city and 
strengthen his own glory, but also to give work to the fragile classes. Located in the center of Paris, the Louvre palace, one of the seats of the power, is, since the reign of Henry IV at least, still under construction. Gathering renowned architects such as Félix Duban, Louis Visconti, and later Hector Lefuel, personally overlooking their work and giving them substantial funding, Napoleon launches in 1853 the completion works of the Louvre, its junction to the Tuileries, and the decoration of its these palaces in the taste of the time. Amongst thousands of documents stored in Archives nationales by the Agence d'architecture du Louvre et des Tuileries, the elevation of the Louvre's facade overlooking the Seine, traced design in watercolour realized under Lefuel's authority, emphasizes the scope of the project and the magnificence that were expected for the banks of the Seine.

Wie sein illustrer Vorgänger träumte der Prinz-Präsident, künftiger Kaiser Napoleon III. davon, in seiner Hauptstadt Paris bedeutsame Spuren zu hinterlassen. Als Ziel hatte er die weitere Verschönerung der Stadt, zwar um seinen eigenen Ruhm zu pflegen, aber auch um den unteren Schichten der Gesellschaft Arbeit zu beschaffen. Der Bau des Louvre-Palastes, einer der Regierungssitze, stand mindestens seit König Heinrich IV. im Herzen von Paris unvollendet. Napoleon rief also die renommierten Architekten zu sich, Félix Duban, Louis Visconti und später Hector Lefuel, denen er seine persönliche Aufmerksamkeit sowie beträchtliche Geldsummen gönnte. So unternahm er schon 1853 die Bauarbeiten zur Vollendung des Louvre, beziehungsweise dessen Verbindung mit den Tuilerien sowie die Verzierung dieser Paläste, dem zeitgenössischen Geschmack gemäß. Unter den tausenden von Dokumenten, die von der Bauagentur des Louvre und der Tuilerien in den Archives nationales abgelegt wurden, befindet sich der Entwurf der Louvre Fassade, die auf die Seine hinausgeht. Diese aquarellierte Zeichnung auf Pauspapier, die unter der Leitung von Lefuel ausgeführt wurde, zeugt von dem Umfang und dem Glanz des Entwurfes einer ansehnlichen Uferfassade zur Seine hin.

\section{AUTEUR}

\section{CÉCILE SOUCHON}

Cécile Souchon, née à Paris en 1946, archiviste paléographe (promotion 1970) a été conservateuradjoint aux archives départementales de Maine-et-loire (1970-1977), directeur des archives départementales de l'Aisne (1977-1993) puis chargée de la section des cartes, plans et photographies aux Archives nationales à Paris (1993-2010). Elle a participé à de nombreux ouvrages sur Laon et sa région, dont Laon : la cathédrale (Zodiaque, 2002, 298 p.) et à diverses publications de l'Inventaire régional de Picardie. Depuis 1973, elle collabore régulièrement aux activités du comité des travaux historiques et scientifiques (CTHS), notamment en tant que membre de la section « Géographie et sciences de l'environnement ». Elle a aussi publié dans la revue du comité français de cartographie, Le Monde des cartes. Elle est enfin spécialiste d'histoire religieuse, comme en témoignent de multiples articles et son ouvrage L'Édit de Nantes (J.-P. Gisserot, 1998, 124 p.) ainsi que sa participation active aux travaux du centre d'études et de recherches prémontrées. 